\title{
Beneficial effects of Jiawei Shenqi-wan and treadmill training on deficits associated with neonatal hypoxic-ischemia in rats
}

\author{
HA NEUI KIM ${ }^{1,2}$, MALK EUN PAK ${ }^{1}$, MYUNG JUN SHIN ${ }^{3,4}$, SOO YEON KIM ${ }^{5,6}$, YONG BEOM SHIN ${ }^{3,4}$, \\ YOUNG JU YUN ${ }^{7}$, HWA KYOUNG SHIN ${ }^{1,2,8}$ and BYUNG TAE CHOI ${ }^{1,2,8}$
}

\begin{abstract}
${ }^{1}$ Department of Korean Medical Science, School of Korean Medicine; ${ }^{2}$ Korean Medical Science Research Center for Healthy-Aging, Pusan National University, Yangsan, Gyeongsangnam-do 50612; ${ }^{3}$ Department of Rehabilitation Medicine, School of Medicine; ${ }^{4}$ Biomedical Research Institute, Pusan National University Hospital, Busan 49241; ${ }^{5}$ Department of Rehabilitation Medicine; ${ }^{6}$ Research Institute for Convergence of Biomedical Science and Technology, Pusan National University Yangsan Hospital; ${ }^{7}$ Department of Integrative Medicine; ${ }^{8}$ Division of Meridian and Structural Medicine, School of Korean Medicine, Pusan National University, Yangsan, Gyeongsangnam-do 50612, Republic of Korea
\end{abstract}

Received April 13, 2016; Accepted January 26, 2017

DOI: $10.3892 /$ etm.2017.4286

\begin{abstract}
Jiawei Shenqi-wan (JSQW), which comprises Shenqi-wan and two additional medicinal herbs, has been widely used for the treatment of various growth impairments, including cerebral palsy. In the present study, JSQW was administered to hypoxic-ischemic Sprague-Dawley rats that underwent treadmill training from 4-7 weeks of age to examine the beneficial effects of combined JSQW and treadmill therapy. Behavioral examinations were performed and a significant improvement in cylinder test performance was observed in rats treated with treadmill training compared with hypoxic-ischemia rats $(\mathrm{P}<0.05)$, as well as a significant improvement in passive avoidance test performance for rats treated with JSQW $(\mathrm{P}<0.05)$. The thickness of the corpus callosum and the integrated optical density (IOD) of myelin basic protein (MBP) were significantly increased by treatment with treadmill therapy alone $(\mathrm{P}<0.01$ and $\mathrm{P}<0.001$, respectively) and treatment with both JSQW and treadmill significantly increased the IOD of MBP compared with hypoxic-ischemia rats $(\mathrm{P}<0.001)$. Western blot analysis revealed that the expression of neuronal nuclei (NeuN) and doublecortin (Dcx)
\end{abstract}

Correspondence to: Professor Byung Tae Choi, Department of Korean Medical Science, School of Korean Medicine, Pusan National University, 49 Busandaehak Street, Yangsan, Gyeongsangnam-do 50612, Republic of Korea

E-mail: choibt@pusan.ac.kr

Abbreviations: JSQW, Jiawei Shenqi-wan; MBP, myelin basic protein; Dcx, doublecortin; BrdU, bromodeoxyuridine; NeuN, neuronal nuclei; GFAP, glial fibrillary acidic protein, mBDNF, mature brain-derived neurotrophic factor; CREB, cAMP response element binding protein; IOD, integrated optical density

Key words: treadmill, Jiawei Shenqi-wan, neonatal hypoxic-ischemia, behavioral function significantly decreased $(\mathrm{P}<0.001$ and $\mathrm{P}<0.05$, respectively) and MBP expression markedly decreased in the ipsilateral subventricular zone of hypoxic-ischemic rats compared with the control group; however, the expression of NeuN was significantly recovered by treatment with both JSQW and treadmill training $(\mathrm{P}<0.05)$. Furthermore, Dcx expression was significantly recovered by treatment with JSQW $(\mathrm{P}<0.05)$, and MBP expression was significantly restored by treatment with treadmill training $(\mathrm{P}<0.01)$. In the immunohistochemical analyses, a significant increase in the number of bromodeoxyuridine (BrdU) positive cells in this region was observed in treadmill-treated rats $(\mathrm{P}<0.05)$, whereas significant increases in the number of Brdu/Dcx or NeuN or glial fibrillary acidic protein double-positive cells were observed only in the group co-treated with JSQW and treadmill $(\mathrm{P}<0.01$, $\mathrm{P}<0.05$ and $\mathrm{P}<0.001$, respectively). These results suggest that JSQW and treadmill training may contribute to behavior recovery following hypoxic-ischemia, and JSQW treatment was particularly effective in promoting memory function via enhancing the differentiation of neuronal progenitor cells. The results of the present study therefore suggest that JSQW may provide an additional treatment option for functional recovery with treadmill training in cerebral palsy.

\section{Introduction}

A common pathogenesis of preterm delivery appears to be perinatal hypoxic-ischemia due to immature lung development, which leads to brain injury (1). The number of babies born preterm is increasing, as are life-long chronic disabilities resulting from hypoxic-ischemia $(2,3)$. Perinatal brain injuries, sensorimotor deficits and impairments in learning and memory, are a major cause of cerebral palsy throughout development and during adulthood (3-5). Treadmill therapy, both full weight-bearing and partial weight-bearing, is a dynamic training approach based on principles that promote movement of the limbs and trunk to generate sensory information through locomotion (6-8). This activity-based strategy improves motor 
and ambulatory skills in children with cerebral palsy and is a frequently used rehabilitation model $(6,7)$.

Shenqi-wan is listed in Dongeuibogam (Treasured Mirror of Eastern Medicine), which was published in 1613 in Korea, and is comprised of seven commonly used medicinal herbs widely prescribed for kidney yang deficiency syndrome $(9,10)$. This herbal preparation has traditionally been used to treat delayed mental and physical development in children $(11,12)$. Shenqi-wan has been shown to have a neuroprotective effect against oxidative stress-induced apoptosis in hippocampal neuronal cells, which suggests that it may be beneficial in the treatment of neurodegenerative disorders (11). Liuwei Dihuang-wan, a preparation similar to Shenqi-wan, has been demonstrated to attenuate neural apoptosis in cerebral injury and improves the synaptic plasticity contributing to cognitive improvement (13-15).

Antler has traditionally been used for the promotion of general health, including the regeneration of bone deficiencies, and has been demonstrated to accelerate physical and neuromotor developmental milestones in young animal $(16,17)$. Jiawei Shenqi-wan (JSQW) is a herbal formula consisting of Shenqi-wan supplemented with deer antler that has been used in Korea in the treatment of growth impairments, including cerebral palsy, to increase the medicinal efficacy of Shenqi-wan $(9,10)$. Medical technological advances have improved the survival rate of premature infants, however there is no definite cure for cerebral palsy. Alternative or complementary therapeutic strategies have previously been investigated to improve upon the available therapies for this disease $(18,19)$. JSQW may have a potentially positive effect by alleviating the symptoms of cerebral palsy.

Treatment with the traditional herbal formulae JSQW with or without treadmill therapy may benefits neurologic endpoints in cerebral palsy $(8,11,13)$; however, little is known about how these treatments modify behaviors in cerebral palsy, particularly for JSQW. In the present study, it was investigated whether JSQW alone or with treadmill therapy induces the recovery of behavioral functions involving motor abnormalities and cognitive deficits in a cerebral palsy-like animal model. The beneficial effects of these treatments and their underlying mechanisms were investigated using a neonatal hypoxic-ischemia rat model. The aim of the present study was to determine whether JSQW and treadmill training have a beneficial effect on hypoxic-ischemia-associated behavioral deficits via recovery of the myelin sheath and cell components, and activation of neuronal progenitor cells. Behavioral testing was performed to assess sensorimotor coordination and memory, and neurogenesis and its related proteins, which are considered to be markers of brain damage associated with hypoxic-ischemic injury.

\section{Materials and methods}

Induction of hypoxic-ischemia model. Pregnant Sprague-Dawley rats at the E17 pregnancy stage $(n=8)$ were obtained from DooYeol Biotech (Seoul, Korea) and housed at $22^{\circ} \mathrm{C}$ and $55 \pm 5 \%$ humidity with a $12 \mathrm{~h}$ light/dark cycle. Rats were fed a commercial diet and provided with water ad libitum throughout the study. All experiments were approved by the Pusan National University (Yangsan, Korea) Animal Care and
Use Committee in accordance with the National Institutes of Health Guidelines (Approval number: PNU-2015-0771). After delivery, 40 pups were selected. Induction of the hypoxic-ischemia model in the pups $(n=32)$ was performed as previously described with modifications (20,21). Briefly, pups were anesthetized under $2 \%$ isoflurane (Choongwae Pharma Corp., Seoul, Korea) at post-natal day 7 (P7; weight, $12-15 \mathrm{~g}$ ) and the left common carotid artery was ligated and subsequently sutured. Following recovery from surgery, rats were placed in a humidified hypoxic chamber with $8 \% \mathrm{O}_{2} / \mathrm{N}_{2}$ mixture for $3 \mathrm{~h}$ in an incubator maintained at $36^{\circ} \mathrm{C}$. Pups were returned to their mother until they were fully weaned, following which they were randomly divided into control, hypoxic-ischemia vehicle (HI), treadmill-treated alone (TM), JSQW-treated alone (JSQW), and co-treated with treadmill and JSQW (TM+JSQW) groups ( $\mathrm{n}=8$ in each) at age P21. Following weaning, mother rats sacrificed by $\mathrm{CO}_{2}$ overdose. Hypoxic-ischemia injuries were indirectly confirmed at P22 via a cylinder test. Body weight and behavioral assessments were monitored from 4 weeks of age (P22), and subsequently at weeks 5, 6, 7, and 8. All treatments were administered under isoflurane anesthesia using a model VIP 3000 calibrated vaporizer (Midmark Corp., Orchard Park, OH, USA).

Preparation of JSQW. JSQW is comprised of nine herbs as follows: $16 \mathrm{~g}$ steamed Rehmannia glutinosa Lib. root, $8 \mathrm{~g}$ Dioscorea batatas Decaisne rhizome, $8 \mathrm{~g}$ Cornus officinalis Sieb. fructus, $6 \mathrm{~g}$ Poria cocos Wolf sclerotium, $6 \mathrm{~g}$ Paeoniasuffruticosa Andr. bark, $6 \mathrm{~g}$ Alisma Canaliculatum rhizome, 6 g Schizandra chinensis Baill. fructus, $6 \mathrm{~g}$ Amomun xanthioides Wallich fructus, and $4 \mathrm{~g}$ Cervus elaphus L. pilose antler from a stag. These medicinal herbs were obtained from the Korean Medicine Hospital (Pusan National University) and authenticated by Professor Dr. Young Ju Yun (Department of Integrative Medicine, School of Korean Medicine, Pusan National University). A voucher specimen was deposited at the Cerebrovascular Diseases Laboratory of Pusan National University. A total of $66 \mathrm{~g}$ JSQW were immersed in 21 distilled water and boiled at $120 \pm 5^{\circ} \mathrm{C}$ for $3 \mathrm{~h}$. The resultant extract was centrifuged $\left(2,000 \times \mathrm{g}\right.$ at $4^{\circ} \mathrm{C}$ for $20 \mathrm{~min}$ ) and filtered through a $0.8-\mu \mathrm{m}$ filter. The filtrate was then concentrated in vacuo for $3 \mathrm{~h}$ at $60 \pm 5^{\circ} \mathrm{C}$ under reduced pressure $(2.3 \mathrm{kPa})$ and converted into a fine dried powder using vacuum drying apparatus with a yield of $12.9 \%(8.52 \mathrm{~g})$. The resulting powder was subsequently dissolved with distilled water for use in further experiments.

Treatment of JSQW and training on treadmill. From P22, doses of $568 \mathrm{mg} / \mathrm{kg}$ JSQW were dissolved with distilled water to final volume $0.3 \mathrm{ml}$ and administered orally for 4 weeks following hypoxic-ischemia in the JSQW and TM+JSQW groups, whereas rats in the control, HI and TM groups were administered distilled water at the same intervals. Rats were trained on a treadmill from P22. In week 4, rats ran at a speed of $5 \mathrm{~m} / \mathrm{min}$ for $10 \mathrm{~min}$ on the first day and gradually progressed to $15 \mathrm{~min}$ on the fifth day. This speed and duration was maintained until the end of the second week. During the next 3 weeks, rats were run with an initial warm-up at $5 \mathrm{~m} / \mathrm{min}$ for $5 \mathrm{~min}$ followed by increasing speed up to 6 and $7 \mathrm{~m} / \mathrm{min}$ for $15 \mathrm{~min}$ and then a recovery period of $5 \mathrm{~m} / \mathrm{min}$ for $5 \mathrm{~min}$. 
Bromodeoxyuridine (BrdU) labeling. BrdU (B5002-5G; Sigma-Aldrich; Merck KGaA, Darmstadt, Germany) is a synthetic thymidine analog that becomes incorporated into a cell's DNA when the cell is dividing during the S-phase of the cell cycle. All rats in the present study were injected with $50 \mathrm{mg} / \mathrm{kg}$ BrdU once daily for 2 weeks (days P22-P35) to label proliferating cells in the subventricular zone of the lateral ventricle in the brain.

Behavioral assessments (cylinder test). The cylinder test was performed to measure the forelimb and assess functional recovery from a unilateral brain lesion. A rat was placed in a glass cylinder $20 \mathrm{~cm}$ in diameter and $30 \mathrm{~cm}$ tall, and the number of limb-wall contacts was counted with ipsilateral (to the lesion site) forelimb and contralateral forelimb support with both forelimbs against the wall and was recorded during lateral exploration. Total count score $=$ (both forelimbs movement-ipsilateral forelimb movement) / total of 20 contacts.

Behavioral assessments (passive avoidance test). The passive avoidance test was used to assess memory function following brain injury using a passive avoidance task (MED-APA-D1; Med Associates Inc. St. Albans, VT, USA). Following 1 day of acclimation to passive avoidance conditions, the rat was placed in the illuminated compartment for the learning trial. Rats who crossed the door into the dark compartment, which then closed automatically, received a single inescapable scrambled foot shock $(0.8 \mathrm{~mA}, 3 \mathrm{sec})$ delivered through the grid base. In this test, the procedure was repeated and a latency time to enter the dark compartment was measured until $180 \mathrm{sec}$. The retention test was performed once in the same manner at $24 \mathrm{~h}$ and 7 days following the learning trial. A latency of $180 \mathrm{sec}$ was assigned to animals that did not enter the dark chamber during the retention test.

Western blot analysis. Rats $(\mathrm{n}=20)$ were anesthetized with $8 \%$ chloral hydrate $(50 \mathrm{mg} / \mathrm{kg}$; C8383-100G; Sigma-Aldrich; Merck KGaA) and sacrificed via intracardial perfusion with saline. Each harvested brain tissue from the subventricular zone and adjacent striatum was washed two times in cold 4-(2-hydroxyethyl)-1-piperazineethansulphonic acid buffer (15-630-080; Thermo Fisher Scientific, Inc., Waltham, MA, USA) and homogenized in lysis buffer consisting of $200 \mathrm{mM}$ Tris (pH 8.0; TRI01; LPS Solution Co., Ltd., Daejeon, Korea), 150 mM NaCl, 2 mM EDTA (ED2SS; Sigma-Aldrich; Merck KGaA), 1 mM NaF (SB0850; Bio Basic, Inc., Amherst, NY, USA), 1\% NP40 (NDB0385; Bio Basic, Inc.), 1 mM phenylmethane sulfonyl fluoride (P7626; Sigma-Aldrich; Merck $\mathrm{KGaA}$ ), 1 mM Na $\mathrm{VO}_{4}$ (S6508; Sigma-Aldrich; Merck KGaA) and protease inhibitor cocktail (P3100-001; GenDEPOT, Inc., Barker, TX, USA) at $4^{\circ} \mathrm{C}$ for $5 \mathrm{~min}$. Equal amounts $(30 \mu \mathrm{g})$ of protein were subsequently separated by $8-12 \%$ SDS-PAGE and resolved proteins were transferred onto a nitrocellulose membrane (Whatman; GE Healthcare Life Sciences, Chalfont, UK), which was subsequently blocked at room temperature with 5\% non-fat milk in Tris-buffered saline containing $0.4 \%$ Tween-20 for $1 \mathrm{~h}$. Membranes were incubated overnight at $4^{\circ} \mathrm{C}$ with primary antibodies (all 1:1,000) against the following: Neuronal nuclei (NeuN; MAB377; EMD Millipore, Billerica, MA, USA), glial fibrillary acidic protein (GFAP; MAB360;
EMD Millipore), Iba-1 (016-20001; Wako Pure Chemicals Industries, Ltd., Osaka, Japan), doublecortin (Dcx; sc-8066; Santa Cruz Biotechnology Inc., Dallas, TX, USA), myelin basic protein (MBP; ab40390; Abcam, Cambridge, MA, USA), mature brain-derived neurotrophic factor (mBDNF; ab75040; Abcam), cAMP response element binding protein (CREB; \#9197; Cell Signaling Technology, Inc., Danvers, MA, USA), and phospho-CREB (pCREB; \#9196; Ser133; Cell Signaling Technology, Inc.). The blots were subsequently incubated at room temperature for $1 \mathrm{~h}$ with the following horseradish peroxidase-conjugated secondary antibodies (all 1:1,000) according to the appropriate species: Goat-anti-mouse (SC-2005; Santa CruzBiotechnology,Inc.), goat anti-rabbit(SC-2004; Santa Cruz Biotechnology, Inc.), donkey anti-goat (SC-2020; Santa Cruz Biotechnology, Inc.), and donkey anti-sheep (A130-100P; Bethyl Laboratories, Inc., Montgomery, TX, USA). Antibody-specific proteins were visualized using an enhanced chemiluminescence detection system (NCI4080KR; Thermo Fisher Scientific, Inc.). $\beta$-actin (1:1,000; a2228; Sigma-Aldrich; Merck KGaA) was used as a loading control for all experiments. Densitometric analysis was performed to quantify immunoreactivity corresponding to the total bands using an Image Quant LAS 4000 system (Fujifilm, Tokyo, Japan). The presented assays are representative of at least three experiments.

Immunohistochemistry. Rats $(\mathrm{n}=20)$ anesthetized with $2 \%$ isoflurane were sacrificed via intracardial perfusion with saline and $4 \%$ paraformaldehyde in PBS. Brains were harvested, post-fixed in the same fixative for $4 \mathrm{~h}$ at $4^{\circ} \mathrm{C}$, and immersed in $30 \%$ sucrose for $48 \mathrm{~h}$ for cryoprotection. Frozen tissues were cut into sections (thickness, $20 \mu \mathrm{m}$ ) and incubated for post-fixation with formaldehyde and blocking with a blocking buffer [1X PBS/5\% normal goat serum (S-1000; Vector Laboratories, Inc., Burlingame, CA, USA) $/ 0.3 \%$ triton X-100] for $1 \mathrm{~h}$ at room temperature. The sections were incubated with primary antibodies against the following: BrdU (1:500; MCA2483; Bio-Rad Laboratories, Inc., Hercules, CA, USA), NeuN (1:500; ABN78; EMD Millipore), GFAP (1:500; AB5804; EMD Millipore), DCX (1:200; sc-8066; Santa Cruz Biotechnology, Inc.), and MBP (1:500; ab40390; Abcam) overnight in PBS at $4^{\circ} \mathrm{C}$. Sections were washed three times with PBS for $10 \mathrm{~min}$ and subsequently incubated with the fluorescein-conjugated horse anti-mouse (1:200; FI-2000; Vector Laboratories, Inc.) and Texas Red-conjugated goat anti-rabbit (1:200; TI-1000; Vector Laboratories, Inc.) secondary antibodies for $2 \mathrm{~h}$ at room temperature in the dark and washed three times with PBS. Slides were mounted in mounting medium (Dako; Agilent Technologies, Inc., Santa Clara, CA, USA) and images were captured using a fluorescence microscope (Carl Zeiss Imager M1; Carl Zeiss AG, Oberkochen, Germany). IMT i-Solution version 10.1 (3122-0770; IMT, Inc., Cicero, NY, USA) was used for automatic measurement of integrated optical density (IOD).

Data analysis. All data are expressed as the mean \pm standard error of the mean. Analysis of variance (ANOVA) was performed using the Sigmastat statistical program version 11.2 (Systat Software, Inc., San Jose, CA, USA). Statistical analysis of data was performed using Student's t-test when comparing 
two groups, or one-way ANOVA via Tukey's post hoc comparison when comparing more than two groups. $\mathrm{P}<0.05$ was considered to indicate a statistically significant difference.

\section{Results}

Effects on functional recovery from hypoxic-ischemia insults. Behavioral analysis was performed to determine the potential benefits of JSQW and treadmill training in hypoxic-ischemia rats. Body weight increased with age and no significant differences were observed among groups (Fig. 1A). A cylinder test was performed for analysis of sensorimotor recovery, and the numbers of cylinder wall contacts with impaired forelimb were found to be significantly decreased in the HI group compared with the control group $(\mathrm{P}<0.05)$; however, significant improvements in the use of the impaired forelimb were observed in the group treated with treadmill training alone at 8 weeks $(\mathrm{P}<0.05)$ and co-treated with JSQW and treadmill at 6 and 8 weeks $(\mathrm{P}<0.05$ and $\mathrm{P}<0.01$, respectively; Fig. $1 \mathrm{~B})$. Retention latency in the passive avoidance test was significantly decreased by hypoxic-ischemia at 7 and 8 weeks $(\mathrm{P}<0.001$ and $\mathrm{P}<0.05$, respectively), whereas a significantly longer latency was observed in rats treated with JSQW $(\mathrm{P}<0.05)$ or co-treated with JSQW and treadmill $(\mathrm{P}<0.01)$ at 8 weeks compared with the HI group (Fig. 1C). These results suggest that JSQW and treadmill training have a significant beneficial effect on memory capabilities and sensorimotor recovery resulting from hypoxic-ischemia injury.

Effects on myelin components and brain recovery-related markers. Morphological and western blot analyses were performed to determine whether functional recovery occurs via myelin components and expression of proteins. The thickness of MBP in the corpus callosum were found to be markedly decreased and the IOD significantly decreased $(\mathrm{P}<0.001)$ in the HI group compared with the control group; however the thickness was significantly recovered in the group treated with treadmill training $(\mathrm{P}<0.01)$. The IOD of MBP in the corpus callosum was also significantly recovered in both the JSQW and treadmill groups $(\mathrm{P}<0.01$ and $\mathrm{P}<0.001$, respectively). The increases in thickness and IOD of MBP were significantly greater in rats treated with treadmill training alone compared with the group treated with JSQW alone $(\mathrm{P}<0.05$ for thickness, $\mathrm{P}<0.001$ for IOD; Fig. 2). Protein expression of cell components and protective markers in the brain were also investigated. The expressions of NeuN and Dcx were significantly decreased in the HI group compared with the control group $(\mathrm{P}<0.001$ and $\mathrm{P}<0.05$, respectively; Fig. 2A-C). However, NeuN expression was significantly recovered by treatment with JSQW or treadmill training $(\mathrm{P}<0.05$ and $\mathrm{P}<0.001$, respectively) and Dcx expression was significantly increased by treatment with JSQW $(\mathrm{P}<0.05)$. Furthermore, the expression of MBP was significantly higher in the treadmill only group compared with the control and HI groups (both $\mathrm{P}<0.01$; Fig. 3). These results suggested that JSQW treatment results in increased expression of Dcx and NeuN, and treadmill training in increased expression of NeuN and MBP, and enhanced thickness and myelin density of the corpus callosum. These changes in protein expression may be associated with the recovery of functional activities from hypoxic-ischemia injury.
Effects on proliferation and differentiation of neuronal progenitor cells. Finally, immunohistochemical analysis was performed to determine whether functional recovery occurs via neurogenesis. The number of cells positive for BrdU was observed to determine levels of neural progenitor cell proliferation. The results demonstrated that there was a significant increase in BrdU positive cells in the treadmill group compared with the HI group $(\mathrm{P}<0.01$ or $\mathrm{P}<0.001$; Figs. 4-6). However, a limited number of cells showed a BrdU/NeuN or Dcx and GFAP double-positive reaction, which is indicative of differentiated cells from neuronal progenitor cells. A significant increase in BrdU/NeuN or Dcx and GFAP double-positive cells compared with the HI group was observed only in the group co-treated with JSQW and treadmill training $(\mathrm{P}<0.05, \mathrm{P}<0.01$ and $\mathrm{P}<0.001$ for BrdU/NeuN, BrdU/Dcx and BrdU/GFAP, respectively; Figs. 4-6). These results suggest that activity-based treadmill training increased the proliferation of neural progenitor cells, and co-treatment with treadmill and JSQW enhanced differentiation to mature neurons and astrocytes.

\section{Discussion}

The results of the present study indicate that both JSQW and treadmill have beneficial therapeutic effects on behavioral deficits of memory function and sensorimotor coordination in a cerebral palsy-like rat model. Treatment with activity-based treadmill training in this animal model was effective in ameliorating forelimb deficits via enhancing myelin components with neuronal protein expression and proliferation of neuronal progenitors. Treatment with JSQW was effective in promoting memory function via enhancing Dcx expression and differentiation of neuronal progenitor cells. With treadmill training, the clinical benefits of JSQW treatment may provide an additional approach for the treatment of memory impairments in patients with cerebral palsy.

The frequency of preterm births has increased and continues to rise due to increased maternal age, the use of fertility treatments, and deleterious environmental factors (1). Premature birth is associated with a high incidence of neurological injury induced by neonatal hypoxic-ischemia (3). Neonatal hypoxic-ischemia is correlated with the subsequent development of cerebral palsy, sensorimotor deficits and impaired learning and memory $(4,5)$. Activity-based strategies, which closely simulate the functional situation of walking, may be the most effective way to achieve successful rehabilitation from neuromuscular disorders. Treadmill exercise is a typical rehabilitation training model and has been shown to yield functional improvements in patients with cerebral palsy $(8,22)$. Following treatment with treadmill training, walking speed and gross motor function have been demonstrated to improve significantly in patients with cerebral palsy (23-25).

Shenqi-wan is comprised of seven medicinal herbs: Rehmannia glutinosa Lib. root, Dioscorea batatas Decaisne rhizome, Cornus officinalis Sieb. fructus, Alisma Canaliculatum All. rhizome, Paeonia suffruticosa Andr. bark, Poria cocos Wolf sclerotium, and Schizandra chinensis Baill. fructus (9-11). JSQW is comprised of nine medicinal herbs; those in Shenqi-wan plus Cervus elaphus L., stag 

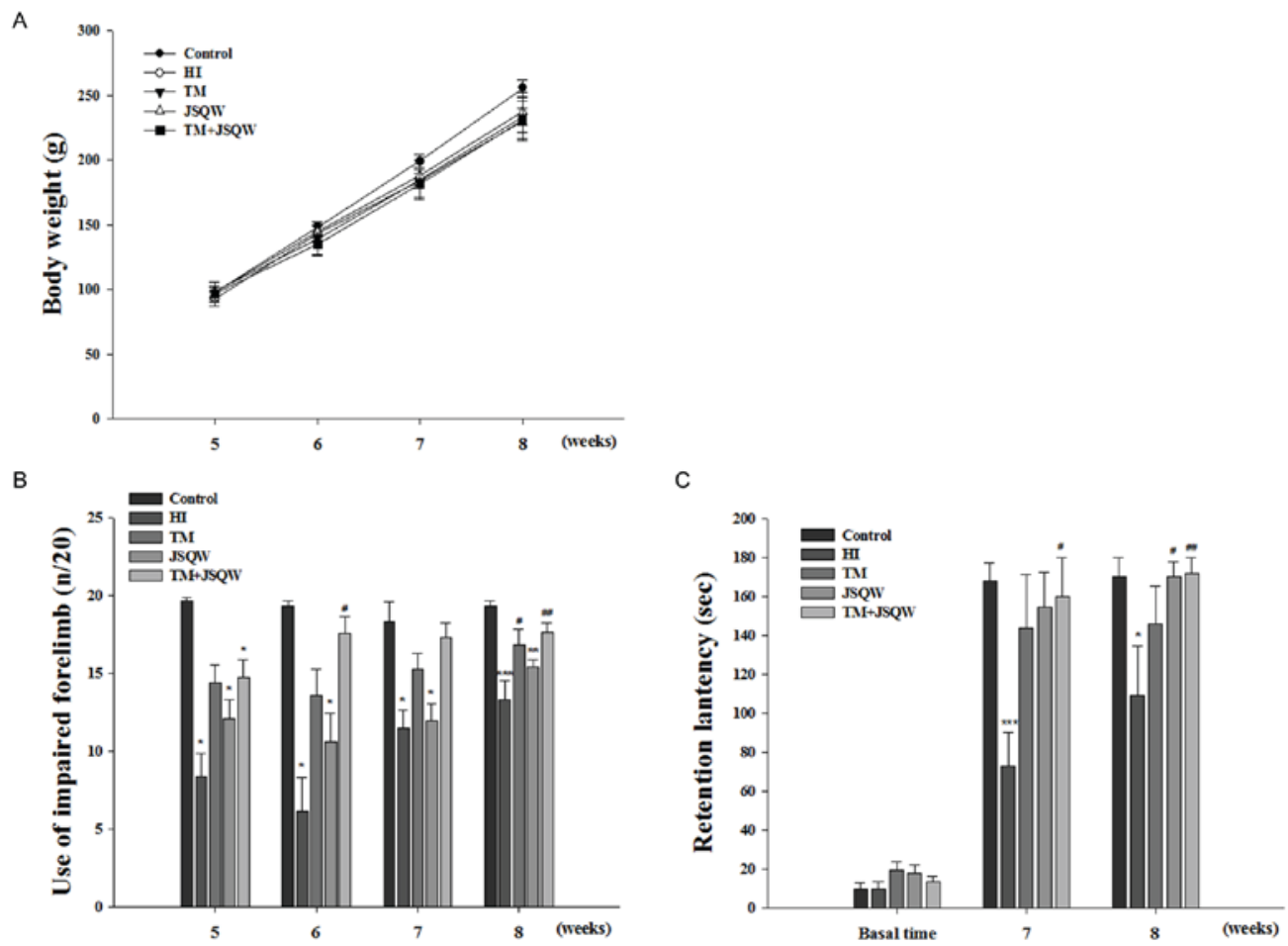

Figure 1. Changes in body weight and results of behavioral tests on the functional deficits caused by HI. (A) Body weight increased with age with no significant differences among groups ( $\mathrm{n}=8$ in each group). Behavioral analysis of (B) cylinder and (C) passive avoidance tests were performed and compared among groups ( $\mathrm{n}=8$ each). The HI group demonstrsted a significant decrease in use of the impaired forelimb and a decrease in retention latency compared with the control group. These deficits were recovered by TM or JSQW treatment. Data are presented as the mean \pm standard error of the mean. ${ }^{*} \mathrm{P}<0.05$, ${ }^{* * *} \mathrm{P}<0.01$ and ${ }^{* * * *} \mathrm{P}<0.001$ vs. control group. ${ }^{\#} \mathrm{P}<0.05$ and ${ }^{\# \#} \mathrm{P}<0.01$ vs. HI group. HI, hypoxic-ischemia; TM, treadmill; JSQW, Jiawei Shenqi-wan.

A

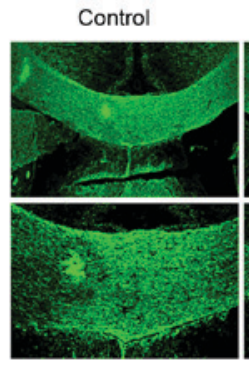

B
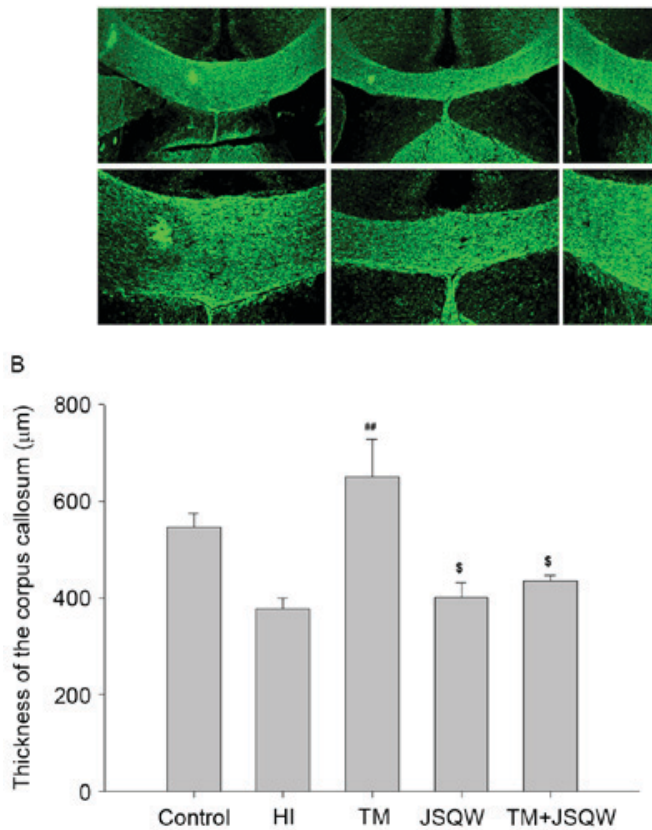

TM

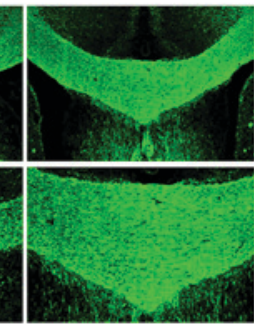

C

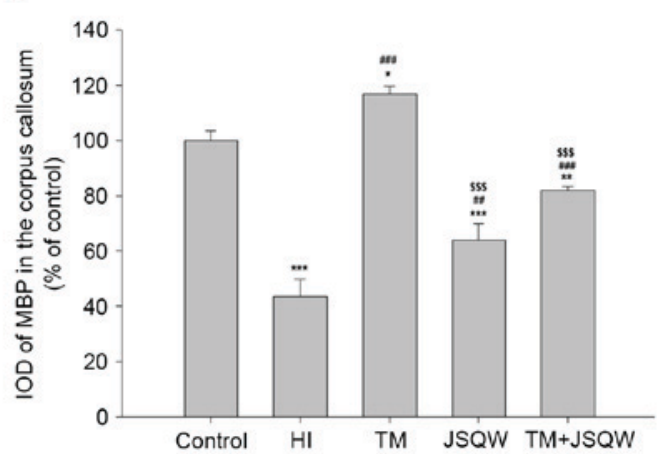

Figure 2. Changes in thickness and myelin component of the corpus callosum. (A) Photomicrography and (B and C) histograms for thickness and IOD of MBP of the corpus callosum. An increase in the thickness and IOD of MBP was observed in the TM group compared with the HI group and the JSQW group. Scale bars $=200 \mu \mathrm{m}$. ${ }^{*} \mathrm{P}<0.05,{ }^{* *} \mathrm{P}<0.01$ and ${ }^{* * * *} \mathrm{P}<0.001$ vs. control group. ${ }^{\# \#} \mathrm{P}<0.01$ and ${ }^{\# \# "} \mathrm{P}<0.001$ vs. HI group. ${ }^{\$} \mathrm{P}<0.05$ and ${ }^{\$ \$ \$} \mathrm{P}<0.001$ vs. TM group. IOD, integrated optical density; MBP, myelin basic protein; TM, treadmill; HI, hypoxic-ischemia; JSQW, Jiawei Shenqi-wan.

pilose antler, and Amomun xanthioides Wallich fructus, and has been demonstrated to have beneficial effects in the treatment of cerebral palsy $(9,10)$. The aim of the present study was to investigate the potentially positive effects of JSQW on alleviation of cerebral palsy impairments using a typical rehabilitation training model. 


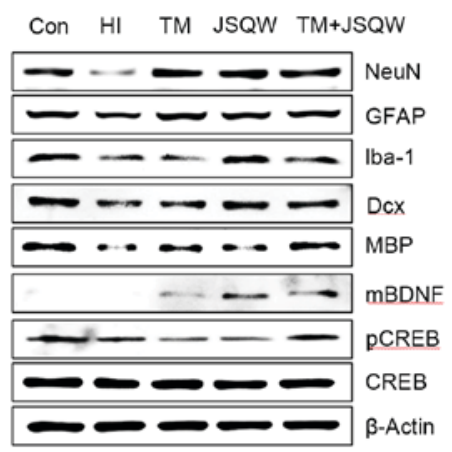

C

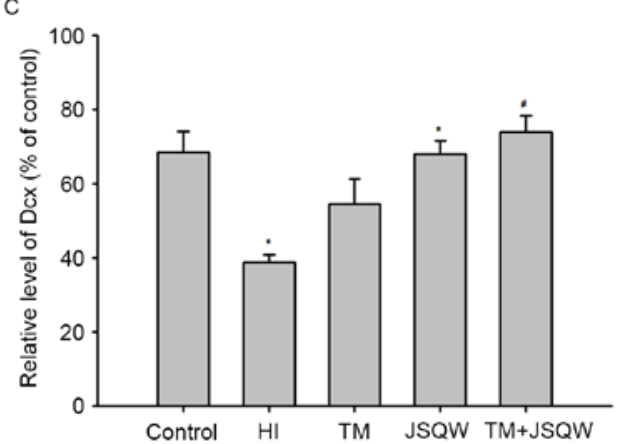

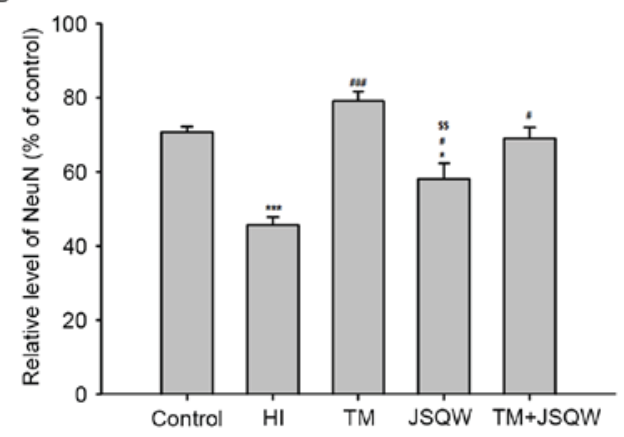

D

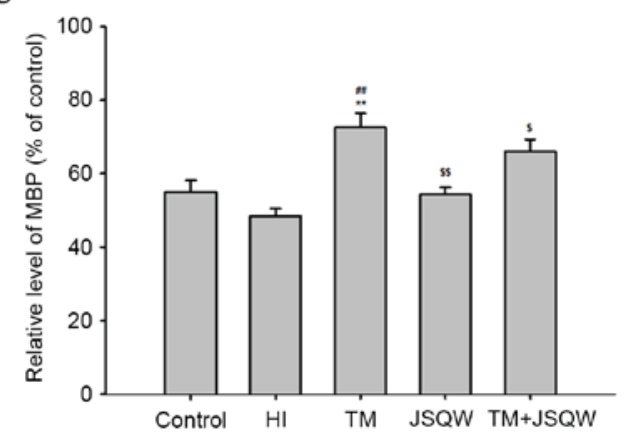

Figure 3. Western blot analysis for functional recovery markers in the ipsilateral subventricular zone of the brain. (A) A typical result from three independent experiments. (B-D) Expressions of NeuN and MBP were increased in the TM group compared with the HI group, and Dcx was increased in the JSQW group. ${ }^{*} \mathrm{P}<0.05,{ }^{* *} \mathrm{P}<0.01$ and ${ }^{* * * *} \mathrm{P}<0.001$ vs. control group. ${ }^{\#} \mathrm{P}<0.05$, ${ }^{\# \#} \mathrm{P}<0.01$ and ${ }^{\# \# \#} \mathrm{P}<0.001$ vs. HI group. ${ }^{\circledR} \mathrm{P}<0.05$ and ${ }^{\$ \$} \mathrm{P}<0.01$ vs. TM group. NeuN, neuronal nuclei; MBP, myelin basic protein; TM, treadmill; HI, hypoxic-ischemia; Dcx, doublecortin; JSQW, Jiawei Shenqi-wan; GFAP, glial fibrillary acidic protein; IBA, ionized calcium-binding adaptor molecule; mBDNF, mature brain-derived neurotrophic factor; $p$, phosphorylated; CREB, cAMP responsive element binding protein.

A
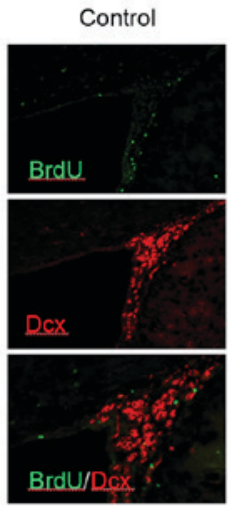

B

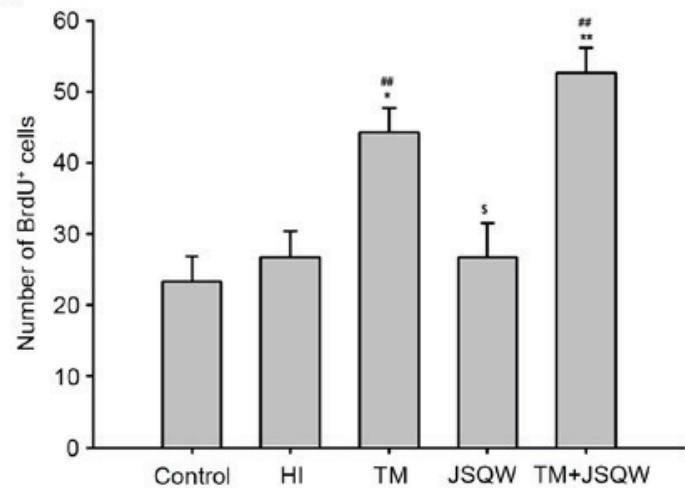

TM
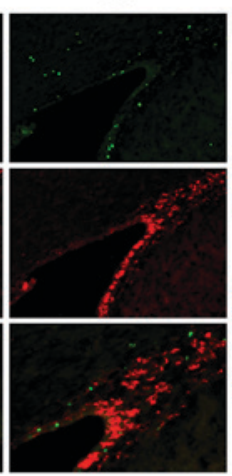

JSQW
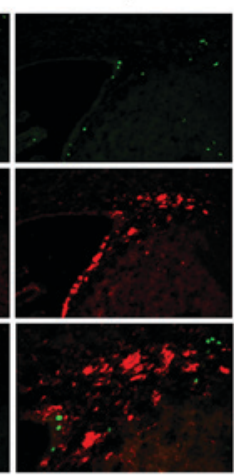

TM+JSQW

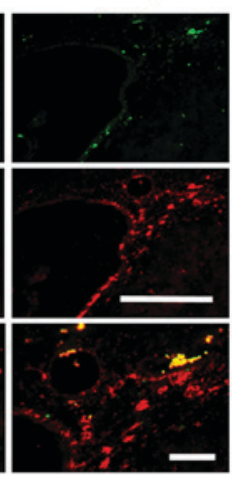

C

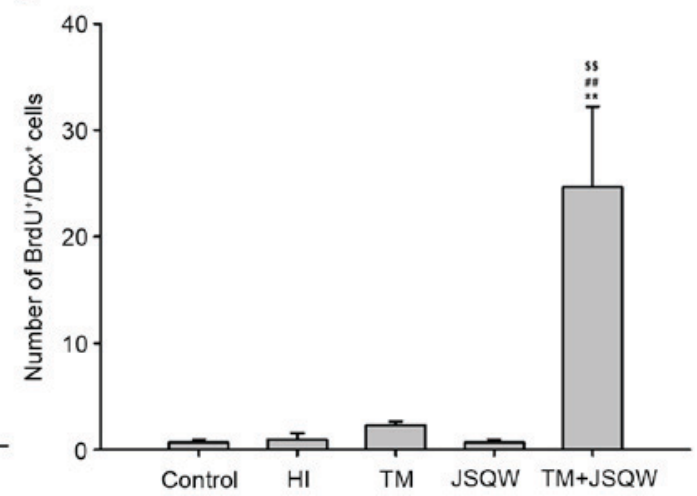

Figure 4. Effect of JSQW and TM treatment on cell proliferation and neuronal differentiation in the subventricular zone of the brain. (A) Photomicrograph and histograms for (B) BrdU positive and (C) BrdU/Dcx double-positive cells. The number of BrdU/Dcx double-positive cells was significantly increased in the TM+JSQW group compared with the HI group. Scale bars $=100 \mu \mathrm{m} .{ }^{*} \mathrm{P}<0.05$ and ${ }^{* *} \mathrm{P}<0.01$ vs. control group. ${ }^{\# \# /} \mathrm{P}<0.01$ vs. HI group. ${ }^{{ }^{\$}} \mathrm{P}<0.05$ and ${ }^{\$ \$} \mathrm{P}<0.01$ vs. TM group. JSQW, Jiawei Shenqi-wan; BrdU, bromodeoxyuridine; Dcx, doublecortin; TM, treadmill; HI, hypoxic-ischemia. 
A
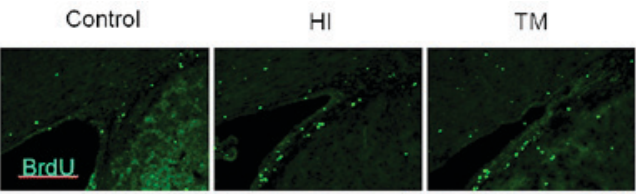

JSQW TM+JSQW
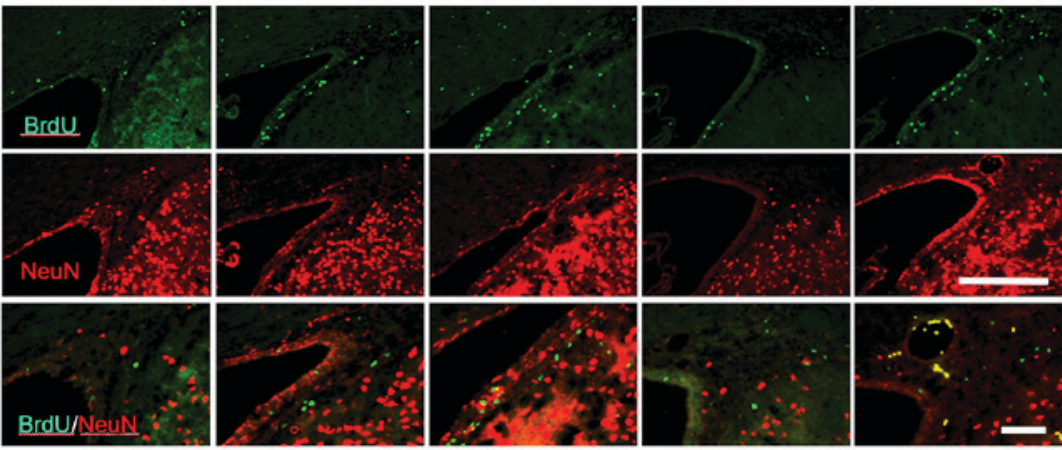

B

C
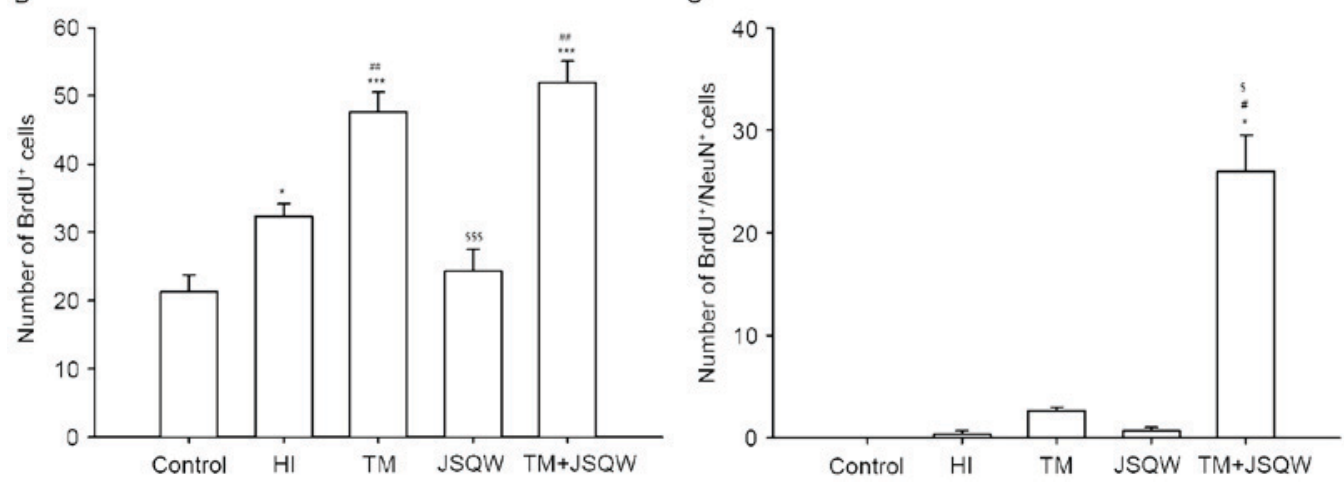

Figure 5. Effect of JSQW and TM treatment on cell proliferation and neuronal maturation in the subventricular zone of the brain. (A) Photomicrograph and histograms for (B) BrdU positive and (C) BrdU/NeuN double-positive cells. The number of proliferated cells was significantly increased in the TM group compared with the HI group, as was the number of mature neuronal cells indicated by BrdU/NeuN double-positive cells in the TM+JSQW group. Scale bars $=100 \mu \mathrm{m}$. ${ }^{*} \mathrm{P}<0.05$ and ${ }^{* * * *} \mathrm{P}<0.001$ vs. control group. ${ }^{\#} \mathrm{P}<0.05$ and ${ }^{\# / *} \mathrm{P}<0.01$ vs. HI group. ${ }^{\$} \mathrm{P}<0.05$ and ${ }^{\$ \$}{ }^{\$ \$} \mathrm{P}<0.001$ vs. TM group. JSQW, Jiawei Shenqi-wan; BrdU, bromodeoxyuridine; NeuN, neuronal nuclei; TM, treadmill; HI, hypoxic-ischemia.

A

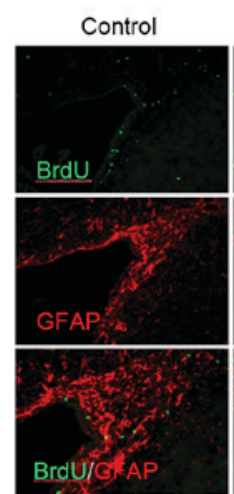

B

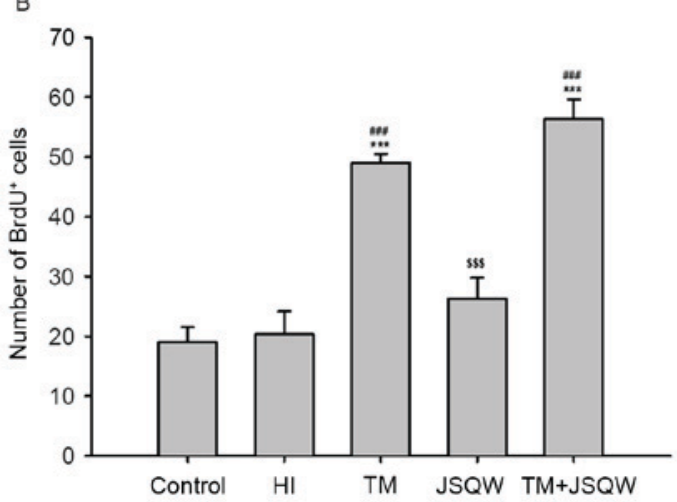

TM

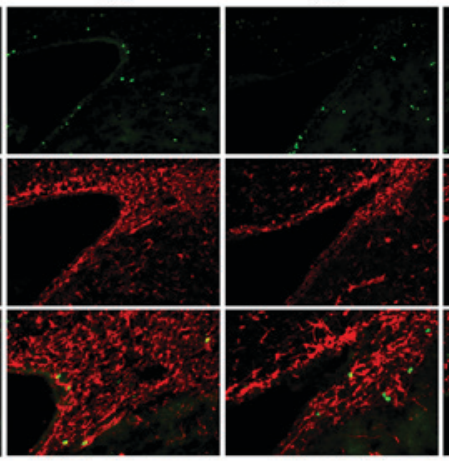

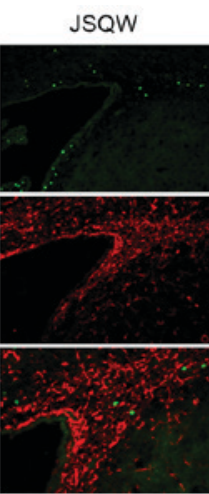

C

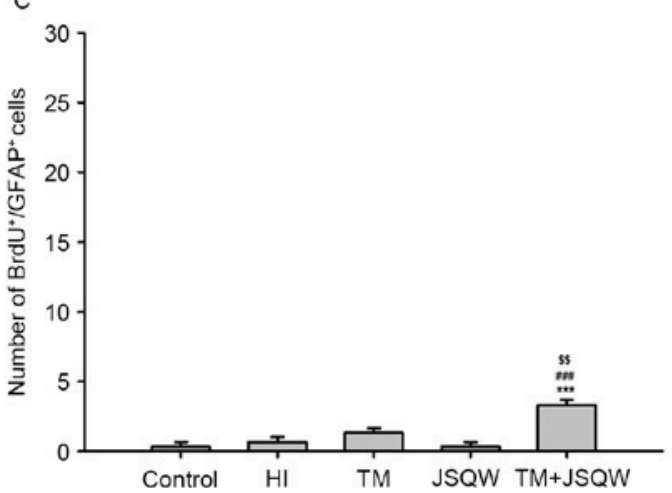

Figure 6. Effect of TM and JSQW treatment on cell proliferation and astrocyte maturation in the subventricular zone of the brain. (A) Photomicrograph and histograms for (B) BrdU and (C) BrdU/GFAP double-positive cells. The number of BrdU/GFAP double-positive cells was significantly increased in the TM+JSQW group compared with the HI group. Scale bars $=100 \mu \mathrm{m}$. ${ }^{* * *} \mathrm{P}<0.001$ vs. control group. ${ }^{\# \# \#} \mathrm{P}<0.001$ vs. HI group. ${ }^{\$ \$} \mathrm{P}<0.01$ and ${ }^{\$ \$ \$} \mathrm{P}<0.001$ vs. TM group. JSQW, Jiawei Shenqi-wan; BrdU, bromodeoxyuridine; GFAP, glial fibrillary acidic protein; TM, treadmill; HI, hypoxic-ischemia. 
Cerebral palsy is characterized by sensorimotor abnormalities and cognitive deficits $(2,5,26)$; therefore, in the present study behavioral testing was performed to compare the outcomes of JSQW and treadmill training. The behavioral data revealed that hypoxic-ischemia affected behavioral performance with sensorimotor coordination and memory, and improvements in behavioral performances were observed with JSQW and treadmill treatment. In particular, treadmill training ameliorated the sensorimotor deficits from hypoxic-ischemia insults and JSQW improved the memory function performance, indicating that JSQW and treadmill training may promote functional recovery via different pathways.

Behavioral deficits are correlated with expression of MBP in white matter $(27,28)$, and so the thickness and expression of myelin in the corpus callosum was measured in the present study. Treadmill training was demonstrated to enhance the thickness and MBP expression of the corpus callosum, which suggests that this training may recover motor sensory activities from hypoxic-ischemia-induced injuries by enhancing myelin components. The results suggest that treadmill training exercise may be an effective therapeutic strategy for alleviating the detrimental effects of cerebral palsy via suppressing hypomyelination in the brain $(29,30)$.

The primary injury sites, which were the subventricular zone and adjacent striatum, in the Vannucci model (20) of hypoxic-ischemia were analyzed by western blotting. Markers for astrocytes and microglia with BDNF and CREB showed no significant changes; however, treatment with treadmill therapy resulted in significantly increased expressions of $\mathrm{NeuN}$ and MBP, whereas treatment with JSQW resulted in an increased expression of Dcx, suggesting promotion of cell component expression. Because neural progenitor cells provide an appropriate therapeutic strategy for neurological disorders, the role of striatal neurogenesis from endogenous progenitor cells was also investigated $(31,32)$.

The results of the present study demonstrate that treadmill training induced significant proliferation of progenitor cells, and co-treatment with JSQW and treadmill training induced an increase in the number of mature functional neurons and astrocytes. This suggests that improvements in behavioral performance induced by treadmill training and JSQW may be derived from neuronal proliferation and differentiation from neuronal progenitor cells.

In Oriental medicine, multi-herb materials, including medicinal plants, animal materials, and minerals, are utilized with the unique guidance of medicine theory (33). There are some preparations similar to Shenqi-wan of Dongeuibogam. Jinkui Shenqi-wan and Jisheng Shenqi-wan are also typically prescribed for treatment of kidney deficiency syndrome $(34,35)$. These traditional herbal formulae consist of different medicinal herbs including Aconitum triphyllum Nakai tuber, Cinnamomum cassia Blume bark and Plantago asiatica L., seed etc. from Shenqi-wan of Dongeuibogam. Liuwei Dihuang-wan consists of the same 6 herbs of Shenqi-wan that are used to treat aging-related functional decline, such as impaired mobility and memory (15). A previous study reported that Shenqi-wan is able to prevent oxidative stress, a primary cause of neuronal death in neurodegenerative disorders (11) and that Liuwei Dihuang-wan ameliorates the deterioration of memory ability (36). These preparations, which have similar combinations to Shenqi-wan of Dongeuibogam, may also be employed in the clinical treatment of growth impairments such as cerebral palsy for a potentially positive effect on alleviating their impairments.

There are no definitive treatments for cerebral palsy available. Treadmill training has been used as a typical rehabilitation model for functional recovery in patients with cerebral palsy (24). However, some patients are unable to employ treadmill walking exercise training due to severe motor abnormalities $(7,25)$. In the present study, some additional therapeutic effects were observed with JSQW treatment, particularly in memory function, via enhancing the differentiation of neuronal progenitor cells. JSQW was found to induce the recovery of memory function resulting from damage and cognitive impairments in hypoxic-ischemic rats. Therefore, treatment with JSQW may offer an additional therapeutic option for the treatment of memory impairment in cerebral palsy and may have clinical applications as a treatment for patients with memory deficits.

\section{Acknowledgements}

The present study was supported by the Convergence of Conventional Medicine and Traditional Korean Medicine R\&D project funded by the Ministry of Health \& Welfare through the Korea Health Industry Development Institute (grant no. HI14C0704).

\section{References}

1. Salmaso N, Jablonska B, Scafidi J, Vaccarino FM and Gallo V: Neurobiology of premature brain injury. Nat Neurosci 17: 341-346, 2014.

2. Yager JY: Animal models of hypoxic-ischemic brain damage in the newborn. Semin Pediatr Neurol 11: 31-46, 2004.

3. Bennet L, Tan S, Van den Heuij L, Derrick M, Groenendaal F, van Bel F, Juul S, Back SA, Northington F, Robertson NJ, et al: Cell therapy for neonatal hypoxia-ischemia and cerebral palsy. Ann Neurol 71: 589-600, 2012

4. van Handel M, Swaab H, de Vries LS and Jongmans MJ: Long-term cognitive and behavioral consequences of neonatal encephalopathy following perinatal asphyxia: A review. Eur J Pediatr 166: 645-654, 2007.

5. Aarnoudse-Moens CS, Weisglas-Kuperus N, van Goudoever JB and Oosterlaan J: Meta-analysis of neurobehavioral outcomes in very preterm and/or very low birth weight children. Pediatrics 124: 717-728, 2009.

6. Richards CL, Malouin F, Dumas F, Marcoux S, Lepage C and Menier C: Early and intensive treadmill locomotor training for young children with cerebral palsy: A feasibility study. Pediatr Phys Ther 9: 158-165, 1997.

7. Cherng RJ, Liu CF, Lau TW and Hong RB: Effect of treadmill training with body weight support on gait and gross motor function in children with spastic cerebral palsy. Am J Phys Med Rehabil 86: 548-555, 2007.

8. Tsai SW, Chen HL, Chang YC and Chen CM: Molecular mechanisms of treadmill therapy on neuromuscular atrophy induced via botulinum toxin A. Neural Plast 2013: 593271, 2013.

9. Natural Products Research Institute of Seoul National University: Treatise on Asian Herbal Medicine. Daewon Publishers Co., Seoul, Vol. I p90, 204, Vol. II p283, 2003.

10. Laboratory of Korean Classical Medical Literature: A translation printed side by side with original Dongeuibogam, A new enlarged edition. Bubin Publishers Co., Seoul, p423: 1255, 2048, 2012.

11. Shin HT, Chung SH, Lee JS, Kim SS, Shin HD, Jang MH, Shin MC, Bahn GH, Paik EK, Park JH and Kim CJ: Protective effect of shenqi-wan against $\mathrm{H}_{2} \mathrm{O}_{2}$-induced apoptosis in hippocampal neuronal cells. Am J Chin Med 31: 675-686, 2003. 
12. Yang J, Wang Y, Bao Y and Guo J: The total flavones from Semen cuscutae reverse the reduction of testosterone level and the expression of androgen receptor gene in kidney-yang deficient mice. J Ethnopharmacol 119: 166-171, 2008.

13. Huang Y, Zhang H, Yang S, Qiao H, Zhou W and Zhang Y: Liuwei Dihuang decoction facilitates the induction of long-term potentiation (LTP) in senescence accelerated mouse/prone 8 (SAMP8) hippocampal slices by inhibiting voltage-dependent calcium channels (VDCCs) and promoting N-methyl-d-aspartate receptor (NMDA) receptors. J Ethnopharmacol 140: 384-390, 2012.

14. Liu JP, Feng L, Zhang MH, Ma DY, Wang SY, Gu J, Fu Q, Qu R and Ma SP: Neuroprotective effect of Liuwei Dihuang decoction on cognition deficits of diabetic encephalopathy in streptozotocin-induced diabetic rat. J Ethnopharmacol 150: 371-381, 2013

15. Wang $\mathrm{P}$, Lv H, Zhang A, Sun H, Yan Gl, Han Y, Wu Xh and Wang $\mathrm{Xj}$ : Improved ultra-performance liquid chromatography with electrospray ionization quadrupole-time-of-flight high-definition mass spectrometry method for the rapid analysis of the chemical constituents of a typical medical formula: Liuwei Dihuang Wan. J Sep Sci 36: 3511-3516, 2013.

16. Chen J, Woodbury MR, Alcorn J and Honaramooz A: Dietary supplementation of female rats with elk velvet antler improves physical and neurological development of offspring. Evid Based Complement Alternat Med 2012: 640680, 2012.

17. Dabrowska N, Kiełbowicz Z, Nowacki W, Bajzert J, Reichert P, Bieżyński J, Zebrowski J, Haczkiewicz K and Cegielski M: Antlerogenic stem cells: Molecular features and potential in rabbit bone regeneration. Connect Tissue Res 57: 539-554, 2015.

18. Damiano DL: Activity, activity, activity: Rethinking our physical therapy approach to cerebral palsy. Phys Ther 86: 1534-1540, 2006.

19. Jones MW, Morgan E and Shelton JE: Primary care of the child with cerebral palsy: A review of systems (part II). J Pediatr Health Care 21: 226-237, 2007.

20. Rice JE III, Vannucci RC and Brierley JB: The influence of immaturity on hypoxic-ischemic brain damage in the rat. Ann Neurol 9: 131-141, 1981

21. Levine S: Anoxic-ischemic encephalophthy in rats. Am J Pathol 36: 1-17, 1960.

22. Wessels M, Lucas C, Eriks I and de Groot S: Body weight-supported gait training for restoration of walking in people with an incomplete spinal cord injury: A systematic review. J Rehabil Med 42: 513-519, 2010.

23. Chrysagis N, Skordilis EK, Stavrou N, Grammatopoulou E and Koutsouki D: The effect of treadmill training on gross motor function and walking speed in ambulatory adolescents with cerebral palsy: A randomized controlled trial. Am J Phys Med Rehabil 91: 747-760, 2012.
24. Willoughby KL, Dodd KJ and Shields N: A systematic review of the effectiveness of treadmill training for children with cerebral palsy. Disabil Rehabil 31: 1971-1979, 2009.

25. Mutlu A, Krosschell K and Spira DG: Treadmill training with partial body-weight support in children with cerebral palsy: A systematic review. Dev Med Child Neurol 51: 268-275, 2009.

26. Eikenes L, Løhaugen GC, Brubakk AM, Skranes J and Håberg AK: Young adults born preterm with very low birth weight demonstrate widespread white matter alterations on brain DTI. NeuroImage 54: 1774-1785, 2011.

27. Cengiz P, Uluc K, Kendigelen P, Akture E, Hutchinson E, Song C, Zhang L, Lee J, Budoff GE, Meyerand E, et al: Chronic neurological deficits in mice after perinatal hypoxia and ischemia correlate with hemispheric tissue loss and white matter injury detected by MRI. Dev Neurosci 33: 270-279, 2011

28. Fan LW, Lin S, Pang Y, Lei M, Zhang F, Rhodes PG and Cai Z: Hypoxia-ischemia induced neurological dysfunction and brain injury in the neonatal rat. Behav Brain Res 165: 80-90, 2005.

29. Hwang DH, Shin HY, Kwon MJ, Choi JY, Ryu BY and Kim BG: Survival of neural stem cell grafts in the lesioned spinal cord is enhanced by a combination of treadmill locomotor training via insulin-like growth factor-1 signaling. J Neurosci 34: 12788-12800, 2014.

30. Kim K, Shin MS, Cho HS and Kim YP: Effects of endurance exercise on expressions of glial fibrillary acidic protein and myelin basic protein in developing rats with maternal infection-induced cerebral palsy. J Exerc Rehabil 10: 9-14, 2014.

31. Abe K, Yamashita T, Takizawa S, Kuroda S, Kinouchi H and Kawahara N: Stem cell therapy for cerebral ischemia: From basic science to clinical applications. J Cereb Blood Flow Metab 32: 1317-1331, 2012.

32. Ohira K, Furuta T, Hioki H, Nakamura KC, Kuramoto E, Tanaka Y, Funatsu N, Shimizu K, Oishi T, Hayashi M, et al: Ischemia-induced neurogenesis of neocortical layer 1 progenitor cells. Nat Neurosci 13: 173-179, 2010.

33. Cheng X, Su X, Chen X, Zhao H, Bo C, Xu J, Bai H and Ning K: Biological ingredient analysis of traditional Chinese medicine preparation based on high-throughput sequencing: The story for Liuwei Dihuang Wan. Sci Rep 4: 5147, 2014.

34. Liu B, Chang Y, Jiang H and Shen B: Extraction of paeonol from Jisheng Shenqi Wan using supercritical fluid extraction. Biomed Chromatogr 21: 79-83, 2007.

35. Xiong X, Wang P, Li X and Zhang Y: Shenqi pill, a traditional Chinese herbal formula, for the treatment of hypertension: A systematic review. Complement Ther Med 23: 484-493, 2015.

36. Wu CR, Lin LW, Wang WH and Hsieh MT: The ameliorating effects of LiuWei Dihuang Wang on cycloheximide-induced impairment of passive avoidance performance in rats. J Ethnopharmacol 113: 79-84, 2007. 